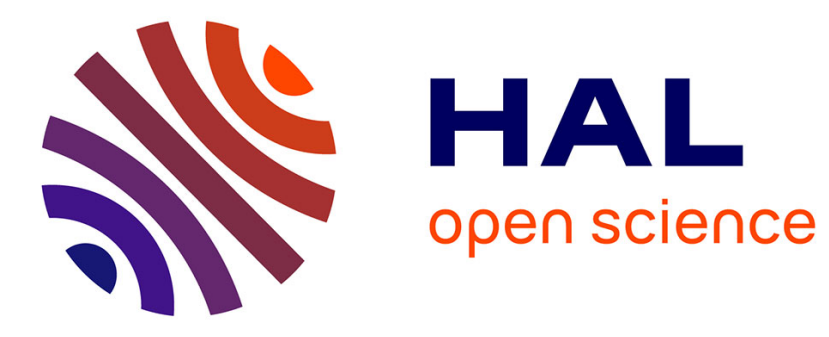

\title{
Recent discrete model for small-signal analysis of traveling-wave tubes
}

\author{
Damien F G Minenna, Artem G Terentyuk, Frédéric André, Yves Elskens, \\ Nikita M. Ryskin
}

\section{- To cite this version:}

Damien F G Minenna, Artem G Terentyuk, Frédéric André, Yves Elskens, Nikita M. Ryskin. Recent discrete model for small-signal analysis of traveling-wave tubes. Physica Scripta, inPress, 10.1088/1402-4896/ab060e. hal-01630803v3

\section{HAL Id: hal-01630803 https://hal.science/hal-01630803v3}

Submitted on 3 Apr 2018

HAL is a multi-disciplinary open access archive for the deposit and dissemination of scientific research documents, whether they are published or not. The documents may come from teaching and research institutions in France or abroad, or from public or private research centers.
L'archive ouverte pluridisciplinaire HAL, est destinée au dépôt et à la diffusion de documents scientifiques de niveau recherche, publiés ou non, émanant des établissements d'enseignement et de recherche français ou étrangers, des laboratoires publics ou privés. 


\title{
Degrees-of-freedom reduction for wave-particle systems : Comparison of Pierce equivalent circuit and recent discrete model for traveling-wave tubes
}

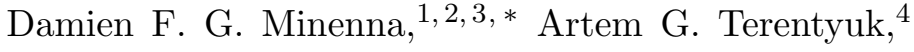

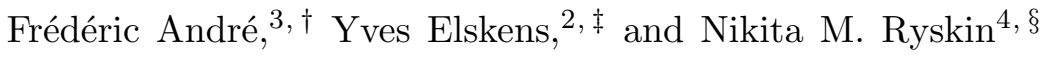 \\ ${ }^{1}$ Centre National d'Études Spatiales, 31401 Toulouse cedex 9, France \\ ${ }^{2}$ Aix-Marseille University, UMR 7345 CNRS PIIM, \\ équipe turbulence plasma, case 322 campus Saint Jérôme, \\ av. esc. Normandie-Niemen, 13397 Marseille cedex 20, France \\ ${ }^{3}$ Thales Electron Devices, rue Latécoère, 2, 78140 Vélizy, France \\ ${ }^{4}$ Saratov State University, 410012 Saratov, Russia, \\ and with Saratov Branch of the Institute of Radio Engineering and Electronics, \\ Russian Academy of Sciences, 410019 Saratov, Russia
}

(Dated: March 29, 2018. Submitted. (CThe authors)

To perform accurate numerical simulations of the wave-particle interaction as it occurs in particular in traveling wave tubes, a new approach using field decomposition with large reduction of degrees of freedom has been proposed : the discrete model. To assess its validity, we compare it with the well-established Pierce equivalent circuit model in small signal regime. Before the comparison, the beam-wave interaction in both models is reformulated using a new pedagogical approach, dealing with associated beam, circuit-beam, and circuit impedances. We show analytically and with a numerical example that the newly developed reduced model is very close to the Pierce model. Interestingly, small deviations do exist at the edges of the amplification band, for which the reduced model offers a physical interpretation.

PACS numbers: 84.40.Fe (Microwave tubes), 52.40.Mj (Particle beam interaction in plasmas)

Keywords: Model reduction, Pierce model, Kuznetsov discrete model, equivalent circuit, dispersion relation, impedances, coupled mode, wave-particle interaction, traveling-wave tube (TWT), time domain, frequency domain, passband, band edge, Gel'fand transform.

\footnotetext{
* Electronic address: damien.minenna@univ-amu.fr

$\dagger$ Electronic address: frederic.andre@thalesgroup.com

‡ Electronic address: yves.elskens@univ-amu.fr

$\S$ Electronic address: ryskinnm@info.sgu.ru
} 


\section{INTRODUCTION}

The interaction of electromagnetic waves and particle beams is an important physical issue in many respects. It relates with foundational aspects of electrodynamics [1, 2], with basic processes in plasmas and accelerators [3 7], as well as with the development of instruments [8] and with industrial applications such as vacuum electronic devices [9-11]. A fully detailed description of this interaction is challenging, because the fields are by definition functions of both space and time, while the many particles require equally many position and velocity variables. Therefore, useful descriptions rely on reducing the dynamical variables to fewer degrees of freedom. For electromagnetic fields, classical reductions include (i) "lumped" variables as used in $R L C$ circuits and nonlinear devices like transistors, diodes, etc..., (ii) finite-elements representations with respect to a mesh in space-time, (iii) Fourier or other representations in terms of given "elementary" or "basis" fields.

The merits of a reduction are assessed by balancing its accuracy and predictive power with the conceptual and computational effort it requires. An extreme example is the reduction of the motion of solid bodies in mechanics to the mere evolution of their centers of mass and Euler angles !

In this work, we compare two model reduction strategies applied to a simple device, the traveling wave tube (TWT). The TWT is a powerful vacuum tube amplifier used notably in space communications, which we further introduce in section III Its first theoretical and efficient description rests on an equivalent circuit representation for the electromagnetic radiofrequency $(\mathrm{RF})$ fields, which is the standard introduction to the device [12, 13. As long as the device is operated in its linear amplification regime, this classical Pierce model in the frequency domain provides a satisfactory description of the dynamics. However, high gain and power being desired, interest in nonlinear regimes calls for the development of new modeling. The more recent discrete model (a.k.a. Kuznetsov discrete model) [14-16] is a promising tool to analyse such regimes beyond the capability of Pierce's model.

The discrete model provides an exact reduction of degrees-of-freedom for electromagnetic fields and allows to build both frequency [17] and time domain algorithms [18, 19] that are faster alternatives to current particle-in-cell (PIC) algorithms [20]. It also offers several new features compared to Pierce's well-known equivalent circuit model, mostly because it is originally expressed in time domain. Examples are drive-induced oscillations where spurious frequencies are generated very far from the drive frequency in the nonlinear regime : this situation will be accessible to simulation 
thanks to this new model. Second, the complex structure of stop bands (frequency or wavenumber ranges for which amplification does not occur) [21] can be accurately described and simulated thus offering a way to progress on the associated oscillation problems. Fundamentally, the discrete model addresses (and originates from) the general situation of periodic, quasi-periodic and chaotic motion of particles interacting with fields, which is of interest to a broad community of physicists and engineers. We outline these aspects in appendices.

Before addressing such more complex situations, the first question is how the new model compares with the existing one in the simplest case of a single carrier operation (i.e. in frequency domain) in the linear regime, the original setting of the Pierce theory [19]. In this article, we show how both models address the issue of coupling two simple systems : the beam and the waves. The resulting four wave theory is a classic theory [22], which is still relevant for both introductory analysis and current R\&D developments.

In section III, we revisit the fluid model for the electron stream. In section IV, we recall the Pierce equivalent circuit model. Additionally, in sections III and IV] we redefine fundamental expressions of beam, wave and circuit impedances, starting with a model involving only space charge fields, and then adding circuit fields. In section $\mathrm{V}$, we present the principles of the discrete model and apply them in the harmonic domain to obtain associated impedances. Finally, we compare both models in section VI, A revisits the sheath helix approximation using the discrete model. B compares the TWT discrete model and beam-plasma models.

\section{TRAVELING-WAVE TUBES}

Traveling-wave tubes (TWTs) are a family of vacuum electron devices, mostly used as microwave power amplifier for radars, electronic warfare and telecommunications since the 1950s, including the majority of space communications since the 1960s [11]. Their main features, compared to other power amplifiers, are their large bandwidth and their excellent power efficiency. They are based on the momentum transfer, similar to kinetic energy transfer, from a beam of electrons to a radio-frequency wave. TWTs are composed of an electron gun, and a slow-wave structure (SWS), as sketched on fig. 1. The radiofrequency electromagnetic wave propagates in the SWS, which is a waveguide designed to propagate the desired RF frequencies with a phase velocity much slower than the celerity of light : this can be achieved in many ways, for instance by winding a metallic wire along a helix with radius $b$ and pitch $d$, so that the wave phase velocity be of the order of $c d / b$ [12, 13, 23]. The beam moves inside the SWS where it interacts with the wave. Resonance occurs 


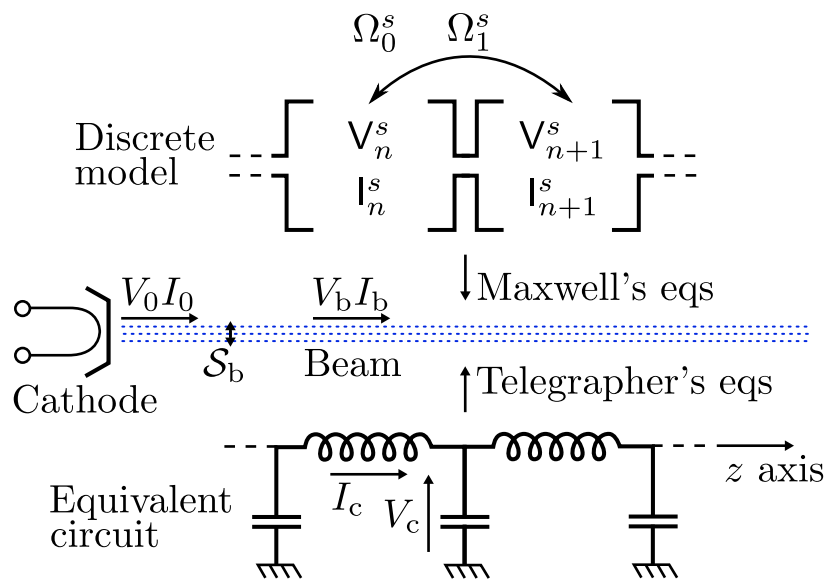

Figure 1. Wave-particles interaction for a periodic slow-wave structure represented using the discrete model (above) and the equivalent circuit (below), along the longitudinal $z$-axis. The beam is assumed to be a weakly perturbed fluid with section area $\mathcal{S}_{\mathrm{b}}$. Variables $V_{\mathrm{c}, \mathrm{b}, 0}$ and $I_{\mathrm{c}, \mathrm{b}, 0}$ are the potential and current for the circuit, beam and cathode (dc beam) respectively. $\mathrm{V}_{n}^{s}$ and $\mathrm{I}_{n}^{s}$ are the temporal variables for electromagnetic circuit fields (see eqs $(29)$ and $(30)$ ) at cell $n$ for the propagation mode $s . \Omega_{m}^{s}$ is the coupling coefficient between cells at range $m$.

when the phase velocity $v_{\text {ph }}$ matches with the beam velocity $v_{0}$. The dispersion relation (hence the phase velocity) and the circuit impedance (determining the coupling efficiency) of the device depend on the geometry of its SWS.

\section{FLUID MODEL FOR THE BEAM}

While both the telegrapher's equation model (section IV) and the discrete model (section V) represent only the waveguide (or circuit) part of a TWT, we devote the current section to the representation of the electron stream, for which a classic fluid model is appropriate. The first hypothesis is that the electrons flux is barely perturbed by the wave presence : we talk about a small signal model, because it cannot afford to study non-linear effects (trapping, chaos) of TWTs and reach important amplifications.

Developments leading to eqs (4) and (11) below are similar to those leading to Louisell's coupled wave system [22] and to the classical analysis by Gilmour [13]. We reformulate them to facilitate the comparison with the discrete model analysis and keep our paper self-contained. In particular, after reaching the dispersion relation of the Pierce equivalent circuit, in the next section, we focus on impedances. A coupled system composed of a beam (b) and a circuit (c) will involve two electric potentials $V_{\mathrm{b}}$ and $V_{\mathrm{c}}$, and two different currents $I_{\mathrm{b}}$ and $I_{\mathrm{c}}$, leading a priori to four 
different impedances, respectively the beam impedance $Z_{\mathrm{b}}$, the circuit-beam impedance $Z_{\mathrm{cb}}$, the beam-circuit impedance $Z_{\mathrm{bc}}$ and the circuit impedance $Z_{\mathrm{c}}$.

\section{A. Space charge waves}

First, we consider the beam without the circuit part. The electron beam is described as a weakly perturbed fluid carrying space charge waves along the longitudinal $z$ coordinate. Timeand space-dependent variables are expressed, according to the space-time Fourier representation, as $F(z, t)=\Re\left(\tilde{F}(\beta, \omega) \mathrm{e}^{-\mathrm{i} \psi}\right)$, with the local phase

$$
\psi=\beta z-\omega t
$$

where $\beta=\omega / v_{\mathrm{ph}}$ is the propagation constant in the longitudinal direction and $\omega$ the wave pulsation for the phase velocity $v_{\mathrm{ph}}$. As one may study non-resonant regimes, one also defines the electronic propagation constant $\beta_{\mathrm{e}}=\omega / v_{0}$, using the beam velocity $v_{0}$. Particle velocities are $v_{0}+\Re\left(\tilde{v} \mathrm{e}^{-\mathrm{i} \psi}\right)$, where the initial electron velocity

$$
v_{0}=\sqrt{2 V_{0} \eta}
$$

is obtained in non-relativistic regime by balancing the kinetic energy $m_{\mathrm{e}} v_{0}^{2} / 2$ with the potential energy $|e| V_{0}$. This initial velocity (2) depends on the cathode (dc beam) potential $V_{0}>0$ and the absolute charge to mass ratio $\eta=|e| / m_{\mathrm{e}}$. Particle charge densities are $\rho_{0}+\Re\left(\tilde{\rho} \mathrm{e}^{-\mathrm{i} \psi}\right)$, with initial density $\rho_{0}=I_{0} /\left(v_{0} \mathcal{S}_{\mathrm{b}}\right)<0$, for a cathode $\left(\mathrm{dc}\right.$ beam) current $I_{0}<0$, and section area of the beam $\mathcal{S}_{\mathrm{b}}$. The electron plasma pulsation of the beam

$$
\omega_{\mathrm{p}}=\sqrt{\eta\left|\rho_{0}\right| / \epsilon_{0}}
$$

is readily expressed in terms of cathode parameters as $\omega_{\mathrm{p}}=\sqrt{\eta\left|I_{0}\right| /\left(v_{0} \mathcal{S}_{\mathrm{b}} \epsilon_{0}\right)}$.

In the linear regime, the relation between perturbed current density and charge density is $\tilde{J}_{z}=\rho_{0} \tilde{v}+v_{0} \tilde{\rho}$. As a first step, we combine this relation with the continuity equation $\partial_{z} J_{z}+\partial_{t} \rho=0$ (viz. i $\beta \tilde{J}_{z}-\mathrm{i} \omega \tilde{\rho}=0$ ), to obtain

$$
\left(\omega-\beta v_{0}\right) \mathcal{S}_{\mathrm{b}} \tilde{J}_{z}=-\omega \frac{\left|I_{0}\right|}{2 V_{0}} \tilde{V}_{\mathrm{b}}
$$

with the perturbed beam potential $\tilde{V}_{\mathrm{b}}=v_{0} \tilde{v} / \eta$. The continuity equation remains unchanged by the presence of circuit waves, so we will keep eq. (4) in the next sections. 
On the other hand, if we only consider space charge waves in our system (neglecting metallic boundary conditions for simplicity), the Euler equation $\left(\partial_{t}+v \partial_{x}\right) v=-\eta E$ for electron motion linearizes to

$$
\left(\mathrm{i} \omega-\mathrm{i} \beta v_{0}\right) \frac{\tilde{v}}{\eta}=-\tilde{E}_{z, \mathrm{sc}},
$$

with the space charge field resulting from the Poisson equation i $\beta \tilde{E}_{z, \mathrm{sc}}=\tilde{\rho} / \epsilon_{0}$. The left hand term in the motion equation (5) is rewritten

$$
\left(\omega-\beta v_{0}\right) \frac{\tilde{v}}{\eta}=\left(\omega-\beta v_{0}\right) \frac{\tilde{V}_{\mathrm{b}}}{v_{0}}
$$

while we express the right hand side of (5) in terms of the current density modulation as $\tilde{E}_{z, \mathrm{sc}}=$ $\mathrm{i} \tilde{J}_{z} /\left(\epsilon_{0} \omega\right)$ thanks to the continuity equation. Introducing the plasma pulsation, the latter expression gives

$$
\mathrm{i} \tilde{E}_{z, \mathrm{sc}}=-\frac{\tilde{J}_{z}}{\epsilon_{0} \omega}=\frac{\omega_{\mathrm{p}}^{2}}{\eta \rho_{0}} \frac{\tilde{J}_{z}}{\omega}=\frac{\omega_{\mathrm{p}}^{2} v_{0}^{2} \mathcal{S}_{\mathrm{b}}}{v_{0} \eta I_{0}} \frac{\tilde{J}_{z}}{\omega}=-\frac{\omega_{\mathrm{p}}^{2}}{\omega v_{0}} \frac{2 V_{0}}{\left|I_{0}\right|} \mathcal{S}_{\mathrm{b}} \tilde{J}_{z}
$$

On the other hand, the relation between the space charge field and the electron current can also be written in terms of a space charge potential modulation $\tilde{V}_{\mathrm{sc}}$, to be related linearly with the beam current modulation $\tilde{I}_{\mathrm{b}}$,

$$
\tilde{E}_{z, \mathrm{sc}}=-\nabla \tilde{V}_{\mathrm{sc}}=\mathrm{i} \beta \tilde{V}_{\mathrm{sc}}=-\mathrm{i} \beta Z_{\mathrm{b}} \tilde{I}_{\mathrm{b}}=-\mathrm{i} \beta Z_{\mathrm{b}} \int_{\mathcal{S}_{\mathrm{b}}} \tilde{J}_{z} \mathrm{~d} x \mathrm{~d} y
$$

on defining the beam characteristic impedance $Z_{\mathrm{b}}$ (with a minus sign due to the negative charge density $\left.\rho_{0}\right)$. Comparing eqs $(7)$ and $(8)$ immediately yields ${ }^{1}$

$$
Z_{\mathrm{b}}(\beta)=\frac{-\omega_{\mathrm{p}}^{2}}{\omega \beta v_{0}} \frac{2 V_{0}}{\left|I_{0}\right|}
$$

as $2 V_{0} /\left|I_{0}\right|=v_{0} /\left(\left|\rho_{0}\right| \eta \mathcal{S}_{\mathrm{b}}\right)$, and if we insert (5) and (6) into (4), and compare with (7), we have the cold ${ }^{2}$ Bohm-Gross dispersion relation [24]

$$
\left(\omega-\beta v_{0}\right)^{2}=\omega_{\mathrm{p}}^{2}
$$

for space charge waves only. They are represented in Fig. 2. The ratio $V_{0} /\left|I_{0}\right|$ is the beam impedance in case of unperturbed beam $\left(\tilde{J}_{z}=\tilde{V}_{\mathrm{b}}=0\right)$, so we refer to it as the cathode (dc) impedance.

\footnotetext{
${ }^{1}$ The minus sign in (4), (8) and (9) comes from our convention $I_{0}<0$. This result reads $Z_{0}=\frac{\omega_{\mathrm{p}}}{\omega} \frac{2 V_{0}}{\left|I_{0}\right|}$ in ref. 22 , where Louisell writes his equation (2.41) in the reference frame of the beam instead of the laboratory frame as here.

${ }^{2}$ In the plasma context, "cold" means neglecting the beam temperature (and pressure) in its ballistic co-moving frame. So it refers to monokinetic beams.
} 


\section{B. Coupling to slow-wave circuits}

Now, we consider the beam coupled with some circuit, and we add circuit waves to the previous

system. In the motion equation (5), the waves simply add to the right-hand side the term $-\tilde{E}_{z, \mathrm{c}}$, corresponding to the electric field from the circuit, and, on combining eqs (4) and (7), we find

$$
\tilde{E}_{z, \mathrm{c}}=-\mathrm{i} \frac{1}{\omega v_{0}}\left[\left(\omega-\beta v_{0}\right)^{2}-\omega_{\mathrm{p}}^{2}\right] \frac{2 V_{0}}{\left|I_{0}\right|} \mathcal{S}_{\mathrm{b}} \tilde{J}_{z}
$$

But this field is equal to

$$
\tilde{E}_{z, \mathrm{c}}=-\nabla \tilde{V}_{\mathrm{c}}=\mathrm{i} \beta \tilde{V}_{\mathrm{c}}=-\mathrm{i} \beta Z_{\mathrm{cb}} \tilde{\mathrm{I}}_{\mathrm{b}}
$$

with the circuit-beam impedance $Z_{\mathrm{cb}}$ corresponding to the response of the circuit potential to the beam current. Hence we find

$$
Z_{\mathrm{cb}}(\beta)=\frac{\left(\omega-\beta v_{0}\right)^{2}-\omega_{\mathrm{p}}^{2}}{\omega \beta v_{0}} \frac{2 V_{0}}{\left|I_{0}\right|} .
$$

At the resonance, where $\beta_{\mathrm{e}}=\beta$ (phase velocity equal to beam velocity), $Z_{\mathrm{cb}}$ acts like the beam impedance as if there were only space charge fields. Since we have here circuit waves, we will recall later the link between eq. (13) and Pierce's circuit impedance (18).

We remark that this treatment of the beam mode is seriously incomplete. Indeed, the plasma reduction factor or the Pierce's space charge parameter $Q C$ [12, 13, 25], which accounts for the tube geometry and is mode-dependent, were totally ignored. We justify this shortcut because our main interest is in the comparison of two circuit theories and not in the beam representation : the circuit-beam impedance (13) will be the same for both circuit theories, irrespectively of this factor.

\section{PIERCE EQUIVALENT CIRCUIT}

Now, we consider the Pierce equivalent circuit model (a.k.a. Pierce's four wave theory, see Fig. 11) provided by [12, 26] in the small signal regime. This frequency model is in fact a combination of the telegrapher's equations for the circuit part and the fluid model of the previous section.

\section{A. Telegrapher's equations}

With the telegrapher's equations, we represent the slow-wave structure as composed of a onedimensional infinite sequence of inductances $L$ and capacitances $C$ per unit length. The evolution 
equations of the circuit potential and current from lossless telegrapher's equations (coupled to the beam current $\left.\tilde{I}_{\mathrm{b}}\right)$ are $[12]$

$$
\begin{aligned}
& -\mathrm{i} \beta \tilde{V}_{\mathrm{c}}=-\mathrm{i} L \omega \tilde{I}_{\mathrm{c}}, \\
& -\mathrm{i} \beta \tilde{I}_{\mathrm{c}}=-\mathrm{i} C \omega \tilde{V}_{\mathrm{c}}+\mathrm{i} \beta \tilde{I}_{\mathrm{b}},
\end{aligned}
$$

with the circuit potential $\tilde{V}_{\mathrm{c}}$, and the circuit current $\tilde{I}_{\mathrm{c}}$. Without beam $\left(\tilde{I}_{\mathrm{b}}=0\right)$, the uncoupled circuit propagation constant is $\beta_{0}=\omega \sqrt{C L}$, and we find $L \omega=Z_{\mathrm{c}} \beta_{0}$ on recalling the classical definition of the characteristic impedance $Z_{\mathrm{c}}=\tilde{V}_{\mathrm{c}} / \tilde{I}_{\mathrm{c}}=\sqrt{L / C}$ which Pierce defines as the circuit impedance. Then we merge the two telegrapher's equations and write the circuit-beam impedance $Z_{\mathrm{cb}}=\tilde{V}_{\mathrm{c}} / \tilde{I}_{\mathrm{b}}$, to find

$$
Z_{\mathrm{cb}}(\beta)=\frac{\beta_{0} \beta}{\beta_{0}^{2}-\beta^{2}} Z_{\mathrm{c}}
$$

We stress that the propagation constant $\beta_{0}$ and the circuit impedance $Z_{\mathrm{c}}$ do not depend on the presence of electrons (cold quantities).

\section{B. Coupling to the fluid model}

In the Pierce equivalent circuit model, one sets eq. (13) equal to eq. (16), leading to

$$
\frac{\left|I_{0}\right|}{2 V_{0}} Z_{\mathrm{c}}=\frac{\left(\beta_{\mathrm{e}}-\beta\right)^{2}-\beta_{\mathrm{p}}^{2}}{\beta_{\mathrm{e}} \beta} \frac{\beta_{0}^{2}-\beta^{2}}{\beta_{0} \beta},
$$

with $\beta_{\mathrm{e}}=\omega / v_{0}$ and $\beta_{\mathrm{p}}=\omega_{\mathrm{p}} / v_{0}$, or in more compact form ${ }^{3}[12$ ]

$$
Z_{\mathrm{c}}=\frac{4 V_{0}}{\left|I_{0}\right|} \mathcal{C}_{\mathrm{p}}^{3}
$$

with $\mathcal{C}_{\mathrm{p}}$ the Pierce coupling (or gain) parameter. Pierce's circuit impedance can also be written equivalently as

$$
Z_{\mathrm{c}}(\beta)=\frac{\left|\tilde{E}_{z, \mathrm{c}}\right|^{2}}{2 \beta^{2}\langle P\rangle}
$$

with $\langle P\rangle$ the harmonic power. Eq. (19) comes directly from $\tilde{V}_{\mathrm{c}} / \tilde{I}_{\mathrm{c}}$, and is used by Pierce to find eq. (18) where the circuit-beam impedances eqs (13) and (16) remain hidden. It would be erroneous to think that for a beamless case $\left(V_{0}=I_{0}=0\right)$, the circuit impedance could be ill-defined

\footnotetext{
${ }^{3}$ In Pierce's theory, waves are sinusoidal in time, as $\mathrm{e}^{-\mathrm{i} \omega t}$, with the space dependence $\mathrm{e}^{\Gamma z}$, and a complex growth rate $\Gamma=\mathrm{i} \beta$ depending on the gain parameter $\mathcal{C}_{\mathrm{p}}$ defined by $17 \mathrm{p}-18$. Three solutions are forward modes, whose propagation constant $\beta_{j}=\mathrm{i} \beta_{\mathrm{e}}-\beta_{\mathrm{e}} \mathcal{C}_{\mathrm{p}} \delta_{j}$ is given by the values of $\delta_{1,2}= \pm \sqrt{3} / 2-\mathrm{i} / 2, \delta_{3}=\mathrm{i}$, while the fourth one has rate $\Gamma_{4}=\mathrm{i} \beta_{4}$, with $\beta_{4}=-\beta_{\mathrm{e}}+\beta_{\mathrm{e}} \mathcal{C}_{\mathrm{p}}^{3} / 4$, thus propagating backwards. While Pierce's development leads rapidly 13 to eq. 18, it bypasses the circuit-beam impedance 13 and 16 needed in our comparison.
} 
: following eq. (19), this is not true. In fact, eq. (18) can only be used for cases with an existing beam as described in section III : the Pierce parameter compensates the effect of the unperturbed beam impedance. This is why the parameter expressing the coupling of the beam with the circuit is the Pierce coupling parameter $\mathcal{C}_{\mathrm{p}}$, not the circuit impedance $Z_{\mathrm{c}}$.

From eq. (17), or on combining eqs (13) and (16), with definition (18), we obtain the "hot" linear dispersion relation

$$
\mathcal{C}_{\mathrm{p}}^{3}=\frac{\left(\omega-\beta v_{0}\right)^{2}-\omega_{\mathrm{p}}^{2}}{2 \omega \beta v_{0}} \frac{\omega^{2}-\beta^{2} v_{\mathrm{ph}, 0}^{2}}{\omega \beta v_{\mathrm{ph}, 0}},
$$

as defined (though written differently) in [12], with $\beta_{\mathrm{e}}=\omega / v_{0}$ and $\beta_{\mathrm{p}}=\omega_{\mathrm{p}} / v_{0}$, and the beamless phase velocity $v_{\mathrm{ph}, 0}=1 / \sqrt{C L}$. This relation exhibits the product of two fractions : one originating from the beam, and the other one from the circuit. It is of the fourth degree, yielding the four natural modes of propagation.

\section{DISCRETE MODEL}

In 1980, Kuznetsov introduced [14] a field decomposition in a periodic medium using Floquet condition and Gel'fand transform. This work was revisited from 2007 on at Saratov University [15, 17], to model coupled cavity traveling wave tubes in frequency domain. This decomposition was then adapted to simulate helix traveling wave tubes by a collaboration [27, 28] between Thales Electron Devices at Vélizy, and Aix-Marseille University, as an alternative to equivalent circuit approaches [29]. Finally renamed discrete model, Kuznetsov's decomposition has been extended [16, 18, 19, 30] to express a self-consistent hamiltonian in a periodic structure with conjugate variables representing electric and magnetic fields.

\section{A. Time domain discrete model}

In this section, we review the basics of the (nonlinear) Kuznetsov discrete theory [14-16]. Electromagnetic fields in three-dimensional Euclidean space are governed by the Maxwell equations with sources, and generate the Lorentz force on particles. We consider the structure as a periodic and uniform waveguide along the $z$ axis with a pitch $d$. Assuming the waveguide to be a perfect metallic conductor, the boundary conditions at the wall are $\mathbf{E}(\mathbf{r}) \times \mathbf{e}_{\perp}=0$, and $\mathbf{H}(\mathbf{r}) \cdot \mathbf{e}_{\perp}=0, \forall \mathbf{r} \in \mathcal{S}_{\mathrm{w}}$, where $\mathcal{S}_{\mathrm{w}}$ is the inner wall surface of the waveguide, and $\mathbf{e}_{\perp}$ is the outgoing unit vector normal to the surface. The important issue about the model is to keep translation invariance along the 
length of the slow wave structure, which enables the Floquet condition. This applies in particular to the helix geometry (see A).

A field propagating in a periodic structure can be expressed with an adapted decomposition called the Gel'fand $\beta$-transform (or Gel'fand mapping) [31], defined as

$$
\mathbf{E}_{\beta}(\mathbf{r}, t)=\sum_{n \in \mathbb{N}} \mathbf{E}\left(\mathbf{r}+n d \mathbf{e}_{z}, t\right) \mathrm{e}^{\mathrm{i} n \beta d}
$$

with $n \in \mathbb{Z}$ labeling the $n^{\text {th }}$ period (or cell) of the structure. The structure itself is an array of copies of a unit cell $\mathcal{V}_{0}$ (with $-d / 2 \leq z \leq d / 2$ for $\mathbf{r} \in \mathcal{V}_{0}$ ). The transform $\mathbf{E}_{\beta}$ definitely exists if the function $\mathbf{E}$ approaches zero sufficiently fast as $\mathbf{r} \rightarrow \pm \infty$. Also, this transform enforces the Floquet condition

$$
\mathbf{E}_{\beta}\left(\mathbf{r}+n d \mathbf{e}_{z}, t\right)=\mathbf{E}_{\beta}(\mathbf{r}, t) \mathrm{e}^{-\mathrm{i} n \beta d}
$$

meaning that translating a field by one period induces a phase-shift $\varphi=\beta d$. In other words, in a periodic system, fields entering a cell are the same when leaving it, up to a phase-shift factor. Expression (21) is invariant by the transformation $\beta \mapsto \beta+2 \pi n^{\prime} / d$ since $\mathrm{e}^{\mathrm{i} 2 \pi n n^{\prime}}=1$, so that $\mathbf{E}_{\beta}=\mathbf{E}_{\beta+2 \pi n^{\prime} / d}$. The inverse $\beta$-transform of 21] is

$$
\mathbf{E}\left(\mathbf{r}+n d \mathbf{e}_{z}, t\right)=(2 \pi)^{-1} \int_{\beta d=-\pi}^{\pi} \mathbf{E}_{\beta}(\mathbf{r}, t) \mathrm{e}^{-\mathrm{i} n \beta d} \mathrm{~d}(\beta d) .
$$

With a view to solving the Maxwell wave equations in the waveguide in the absence of a beam ("cold" fields), we search for solutions by means of the usual separation of variables, with harmonic dependence on time in the form $\mathrm{e}^{\mathrm{i} \Omega t}$. Applying the $\beta$-transform 21) to Maxwell equations then leads to the Helmholtz linear system, with eigenvalues $\Omega_{\beta}^{s}$,

$$
\begin{aligned}
& \operatorname{rot} \mathbf{E}_{\beta}^{s}(\mathbf{r})=-\mathrm{i} \mu_{0} \Omega_{\beta}^{s} \mathbf{H}_{\beta}^{s}(\mathbf{r}), \\
& \operatorname{rot} \mathbf{H}_{\beta}^{s}(\mathbf{r})=\mathrm{i} \epsilon_{0} \Omega_{\beta}^{s} \mathbf{E}_{\beta}^{s}(\mathbf{r}),
\end{aligned}
$$

where propagation modes $\mathbf{E}_{\beta}^{s}$ and $\mathbf{H}_{\beta}^{s}$ are (solenoidal) eigenvector electromagnetic fields, satisfying the Floquet condition $(22)$ and wall boundary conditions on $\partial \mathcal{V}_{0}$. As the Helmholtz operator is hermitian, they constitute a basis for fields, and we introduce $\mathrm{V}_{\beta}^{s}(t), \mathbf{I}_{\beta}^{s}(t) \in \mathbb{C}$ as the discretized set of field generalized coordinates :

$$
\begin{aligned}
& \mathbf{E}_{\beta}(\mathbf{r}, t)=\sum_{s \in \mathbb{Z}} \mathrm{V}_{\beta}^{s}(t) \mathbf{E}_{\beta}^{s}(\mathbf{r}), \\
& \mathbf{H}_{\beta}(\mathbf{r}, t)=\sum_{s \in \mathbb{Z}} \mathrm{il}_{\beta}^{s}(t) \mathbf{H}_{\beta}^{s}(\mathbf{r}),
\end{aligned}
$$


with an arbitrary factor $\mathrm{i}$ in the second equation to ensure later that physical values of the model will be pure real ones. Those relations are valid in the reference cell $\mathcal{V}_{0}$, but all functions satisfy the Floquet condition, so they hold everywhere. Eigenfields $\mathbf{E}_{\beta}^{s}$ and $\mathbf{H}_{\beta}^{s}$, respectively for the electric and the magnetic field, satisfy the normalization ${ }^{4}$

$$
N_{\beta}^{s} \delta_{s^{\prime}}^{s}=\int_{\mathcal{V}_{0}} \epsilon_{0} \mathbf{E}_{\beta}^{s} \cdot \mathbf{E}_{\beta}^{s^{\prime *}} \mathrm{~d}^{3} \mathbf{r}=\int_{\mathcal{V}_{0}} \mu_{0} \mathbf{H}_{\beta}^{s} \cdot \mathbf{H}_{\beta}^{s^{\prime} *} \mathrm{~d}^{3} \mathbf{r}
$$

where $\mathcal{V}_{0}$ is the cell volume, and $\delta_{s^{\prime}}^{s}$ is the Kronecker symbol.

The elegant transform (21) is based on Fourier series and shares many of their properties. In particular, the transform of a product is the convolution of the transforms of its factors. Applying this property to eq. (26) completes our initial search for a discrete model :

$$
\mathbf{E}(\mathbf{r}, t)=\sum_{s \in \mathbb{N}} \sum_{n \in \mathbb{Z}} \mathbf{V}_{n}^{s}(t) \mathbf{E}_{-n}^{s}(\mathbf{r})
$$

with $\mathrm{V}_{n}^{s}$ the Gel'fand transform of $\mathrm{V}_{\beta}^{s}$. These are the discrete variables determining the electric field. The magnetic field is also discretized 5 with its own coordinates $\mathbf{I}_{n}^{s}$

$$
\mathbf{H}(\mathbf{r}, t)=\mathrm{i} \sum_{s \in \mathbb{N}} \sum_{n \in \mathbb{Z}} \mathrm{I}_{n}^{s}(t) \mathbf{H}_{-n}^{s}(\mathbf{r}) .
$$

Note the i factor needed to have real $I_{n}^{s}$ variables instead of purely imaginary one.

The interest of this decomposition appears in eqs (29)-(30). For a single propagating mode, there are $2 n_{\max }$ different time variables (viz. degrees of freedom) for the fields in a delay-line with $n_{\max }$ periods. In comparison, finite difference techniques used in particle-in-cell codes necessitate several millions of degrees of freedom to obtain the same accuracy, because finite differences sample the field on their mesh, whereas the Gel'fand reduction uses adapted field shapes $\mathbf{E}, \mathbf{H}$.

We now introduce the beam. Using Maxwell equations with sources, the field decompositions (29)- (30), and the Helmholtz equations (24)-25), we find the evolution equations [16]

$$
\begin{aligned}
-\sum_{s \in \mathbb{N}} \mathbf{l}_{\beta}^{s} \Omega_{\beta}^{s} \mathbf{E}_{\beta}^{s} & =\sum_{s \in \mathbb{N}} \frac{\partial \mathrm{V}_{\beta}^{s}}{\partial t} \mathbf{E}_{\beta}^{s}+\frac{\mathbf{J}_{\beta}}{\epsilon_{0}}-\frac{\partial \nabla \phi_{\beta}}{\partial t}, \\
\sum_{s \in \mathbb{N}} \mathbf{V}_{\beta}^{s} \Omega_{\beta}^{s} \mathbf{H}_{\beta}^{s} & =\sum_{s \in \mathbb{N}} \frac{\partial \mathbf{l}_{\beta}^{s}}{\partial t} \mathbf{H}_{\beta}^{s},
\end{aligned}
$$

where $\mathbf{J}(\mathbf{r}, t)$ is the $3 \mathrm{D}$ charge density, and the space-charge potential $\phi(\mathbf{r}, t)$ satisfies the Poisson equation $\Delta \phi=-\rho / \epsilon_{0}$.

\footnotetext{
${ }^{4}$ In [16], this normalisation is chosen equal to the eigenfield pulsation $\Omega_{\beta}^{s}$ so that the canonical variables of the Hamiltonian (not discussed here) are the field coefficients $\mathrm{V}_{n}^{s}$ and $\mathrm{I}_{n}^{s}$ in $29-(30)$; their dimension is then the square root of an action. In [15, this normalisation has the dimension of an energy, and $\mathrm{V}_{n}^{s}$ and $\mathrm{I}_{n}^{s}$ become dimensionless.

${ }^{5}$ Ref. [15] uses $\mathrm{V}_{\beta}^{s}=-\mathrm{I}_{\beta}^{s}$ but this is misleading [32]. We also use $-\pi \leqslant \beta d \leqslant \pi$ instead of $0 \leqslant \beta d \leqslant 2 \pi$.
} 


\section{B. Harmonic domain discrete model}

In small signal regime, the discrete model in harmonic domain couples the charge density $\mathbf{J}(\mathbf{r}, t)=\mathbf{J}_{0}+\Re\left(\tilde{\mathbf{J}}(\mathbf{r}) \mathrm{e}^{\mathrm{i} \omega t}\right)$ with temporal variables $\mathrm{V}_{\beta}^{s}(t)=\tilde{\mathbf{V}}_{\beta}^{s} \mathrm{e}^{\mathrm{i} \omega t}$ and $\mathbf{I}_{\beta}^{s}(t)=\tilde{\mathbf{I}}_{\beta}^{s} \mathrm{e}^{\mathrm{i} \omega t}$. From eq. (32) and thanks to the eigenfields orthogonality, we have $\tilde{\mathbf{I}}_{\beta}^{s}=-\mathrm{i} \Omega_{\beta}^{s} \tilde{\mathrm{V}}_{\beta}^{s} / \omega$, so the evolution equation (31) becomes

$$
\sum_{s \in \mathbb{N}} \frac{\left(\Omega_{\beta}^{s}\right)^{2}-\omega^{2}}{\omega} \tilde{\mathbf{V}}_{\beta}^{s} \mathbf{E}_{\beta}^{s}(\mathbf{r})=\frac{-\mathrm{i}}{\epsilon_{0}} \tilde{\mathbf{J}}_{\beta}(\mathbf{r})-\omega \nabla \tilde{\phi}_{\beta}(\mathbf{r}),
$$

where the space charge term $\nabla \tilde{\phi}_{\beta}$ will disappear under integration over the cell volume thanks to boundary conditions [16]. We dot-multiply eq. (33) by the complex conjugate $\mathbf{E}_{\beta}^{s *}$ and integrate over space (viz. we project on the mode $(s, \beta)$ ), to find (for a beam with uniform section and small radius)

$$
\frac{\left(\Omega_{\beta}^{s}\right)^{2}-\omega^{2}}{\omega} \tilde{\mathrm{V}}_{\beta}^{s}=-\mathrm{i} S_{\mathrm{b}} \int_{0}^{d} \tilde{J}_{z, \beta}(z) \mathrm{F}_{z, \beta}^{s *}(z) \mathrm{d} z,
$$

with $\mathrm{F}_{z, \beta}^{s *}(z)=\mathrm{E}_{z, \beta}^{s *}(z) / N_{\beta}^{s}$ related to the vector potential eigenfunction. Eq. (34) from Maxwell equations replaces the telegrapher's equations in the discrete model. We mainly deal with eigenmodes off resonance, so $\Omega_{\beta}^{s} \neq \omega$ generally.

To complete our model, we take the same weakly perturbed electron beam as in section IV. leading to the same linear equation (11) but this time with the circuit field (29) and the charge density spatially modulated in $z$ by $\tilde{J}_{z}(z)=\hat{J}_{z} \mathrm{e}^{-\mathrm{i} \beta z}$. Then using the inverse Fourier transform, we obtain

$$
\begin{aligned}
\tilde{J}_{z, \beta^{\prime}}(z) & =\sum_{n \in \mathbb{Z}} \tilde{J}_{z}(z+n d) \mathrm{e}^{\mathrm{i} n \beta^{\prime} d}=\sum_{n \in \mathbb{Z}} \hat{J}_{z} \mathrm{e}^{-\mathrm{i} \beta z+\mathrm{i}\left(\beta^{\prime}-\beta\right) n d} \\
& =\hat{J}_{z} \mathrm{e}^{-\mathrm{i} \beta z} \sum_{p \in(2 \pi / d) \mathbb{Z}} \delta\left(\frac{\beta^{\prime}-\beta-p}{2 \pi} d\right)
\end{aligned}
$$

for any wave number $\beta^{\prime}$, where $\delta$ is Dirac's distribution. The same is performed for the electric field coefficient, $\tilde{\mathrm{V}}_{\beta^{\prime}}^{s}=\sum_{p} \hat{\mathrm{V}}_{p}^{s} \delta\left(\beta^{\prime}-\beta-p\right) \frac{2 \pi}{d}$, and $(34)$ becomes

$$
\frac{\left(\Omega_{\beta+p}^{s}\right)^{2}-\omega^{2}}{\omega} \hat{\mathrm{V}}_{p}^{s}=-\mathrm{i} S_{\mathrm{b}} \hat{J}_{z} \int_{-d / 2}^{d / 2} \mathrm{e}^{-\mathrm{i}(\beta+p) z} \mathrm{~F}_{z, \beta+p}^{s *}(z) \mathrm{d} z .
$$

In the circuit field (29), the integration on $\beta d$ ranges only over $[-\pi, \pi]$, so the sum reduces to the single term $p=0$ (indeed, only one band, $s=0$, matters for the waves), and we drop subscript $p$. As the Gel'fand eigenfield must respect $\mathrm{E}_{z, \beta}^{s}(z)=\hat{\mathrm{E}}_{z}^{s} \mathrm{e}^{-\mathrm{i} \beta z}$ (and using $\left.\mathrm{F}_{z, \beta}^{s *}(0,0, z)=\hat{\mathrm{E}}_{z}^{s *} \mathrm{e}^{\mathrm{i} \beta z} / N_{\beta}^{s}\right)$, we have the perturbed circuit field

$$
\tilde{E}_{z, \mathrm{c}}=\hat{\mathrm{V}}^{s} \mathrm{E}_{z, \beta}^{s}(z) \mathrm{e}^{\mathrm{i} \beta z}=\hat{\mathrm{V}}^{s} \hat{\mathrm{E}}_{z}^{s}
$$


So we finally reach a new expression for 36

$$
\frac{\left(\Omega_{\beta}^{s}\right)^{2}-\omega^{2}}{\omega} \hat{\mathrm{V}}^{s}=-\mathrm{i} S_{\mathrm{b}} \hat{J}_{z} d \frac{\hat{E}_{z}^{s *}}{N_{\beta}^{s}},
$$

and we rewrite eq. (11) with eqs $(35)$ and (37).

On the other hand, we can rewrite the circuit impedance from the discrete model as [19]

$$
Z_{\mathrm{c}}(\beta)=\frac{\left|\mathrm{E}_{z, \beta}^{s}(r=0)\right|^{2} d}{\beta^{2} v_{\mathrm{g}} N_{\beta}^{s}},
$$

where $v_{\mathrm{g}}(s, \beta)$ is the group velocity, and we can compare eq. (39) to the equivalent circuit impedance (19). We remark that this wave impedance tends to infinity at the passband edges where the group velocity vanishes. An advantage of $(39)$ is that it involves only experimentally known cold values, providing values for $\mathrm{E}_{z, \beta}^{s}(r=0)$ (and its values in the $n$-representation) from $Z_{\mathrm{c}}$. Following the definition (8), but for the circuit field and the circuit-beam impedance, we rewrite the latter for the discrete model

$$
Z_{\mathrm{cb}}(\beta)=-\frac{\mathrm{i}}{\beta} \frac{\hat{\mathrm{V}}^{s} \hat{\mathrm{E}}_{z}^{s}}{S_{\mathrm{b}} \hat{J}_{z}}
$$

for a beam with uniform section. We insert this relation in eq. (38) and use eq. (39) to find a new expression

$$
Z_{\mathrm{cb}}(\beta)=\frac{\omega \beta v_{\mathrm{g}}}{\omega^{2}-\left(\Omega_{\beta}^{s}\right)^{2}} Z_{\mathrm{c}}
$$

enabling us to compare the equivalent circuit-beam impedance 13 with the circuit impedance (18). Substituting eqs 13 and (41) in (18), the "hot" linear dispersion relation of the discrete model becomes

$$
\mathcal{C}_{\mathrm{p}}^{3}=\frac{\left(\omega-\beta v_{0}\right)^{2}-\omega_{\mathrm{p}}^{2}}{2 \omega \beta v_{0}} \frac{\omega^{2}-\left(\Omega_{\beta}^{s}\right)^{2}}{\omega \beta v_{\mathrm{g}}} .
$$

Even if they are similar, relations $(20)$ and $(42)$ are not identical.

\section{COMPARISON}

To compare accurately both models from sections $\left[\mathrm{IV}\right.$ and $\mathrm{V}$, we take a phase velocity $v_{\mathrm{ph}, 0}$ depending on our "cold" dispersion relation, instead of taking it constant, as in Pierce's theory. 


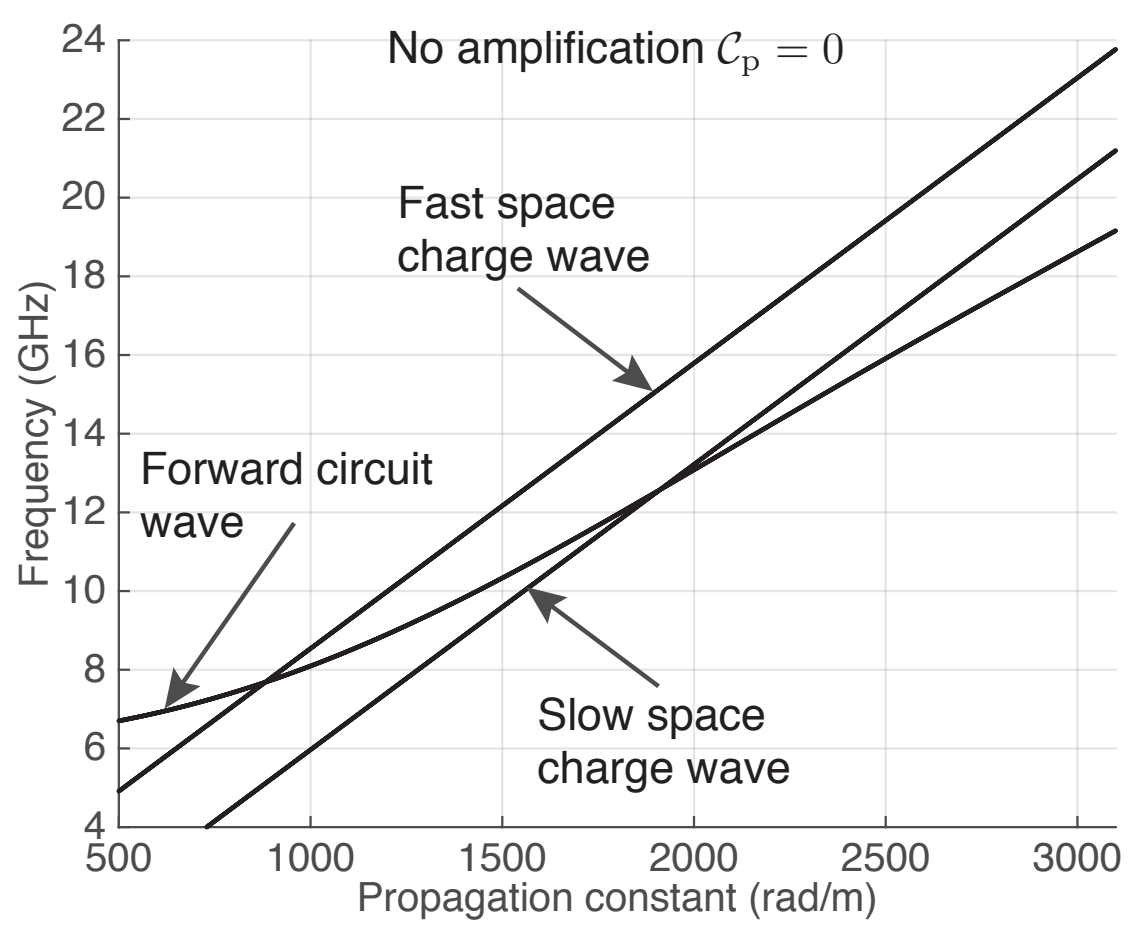

Figure 2. Dispersion diagrams without coupling $\left(\mathcal{C}_{\mathrm{p}}=0\right.$ as if Pierce circuit impedance tends to zero $)$ for the equivalent circuit model (eq. (20)), or equivalently for the harmonic domain discrete model (42). Solutions are purely real values. The forward circuit wave is simply the "cold" dispersion relation 10.

\section{A. Without amplification}

We first consider the case when the Pierce parameter tends to zero $\left(\mathcal{C}_{\mathrm{p}} \rightarrow 0\right)$. The four solutions of the dispersion relations (20) and (42) of the equivalent circuit and discrete models are identical. Solutions for forward and return circuit waves are $\omega= \pm \beta v_{\mathrm{ph}, 0}$. For the discrete model, we have $\omega= \pm \Omega_{\beta}^{s}$ but because we take the same "cold" dispersion relation and because $\mathcal{C}_{\mathrm{p}} \rightarrow 0$, we can take $\Omega_{\beta}^{s}=\beta v_{\mathrm{ph}, 0}$, leading to identical results for both models. Solutions for the slow and fast beam waves are $\omega=\beta v_{0} \pm \omega_{\mathrm{p}}$. Those solutions are presented in Fig. 2 .

\section{B. With amplification}

Taking now the beam-mode coupling into account $\left(\mathcal{C}_{\mathrm{p}}>0\right)$, we notice that relations 20 and 42 coincide when using the first order linear approximation for numerators of the second fractions. 


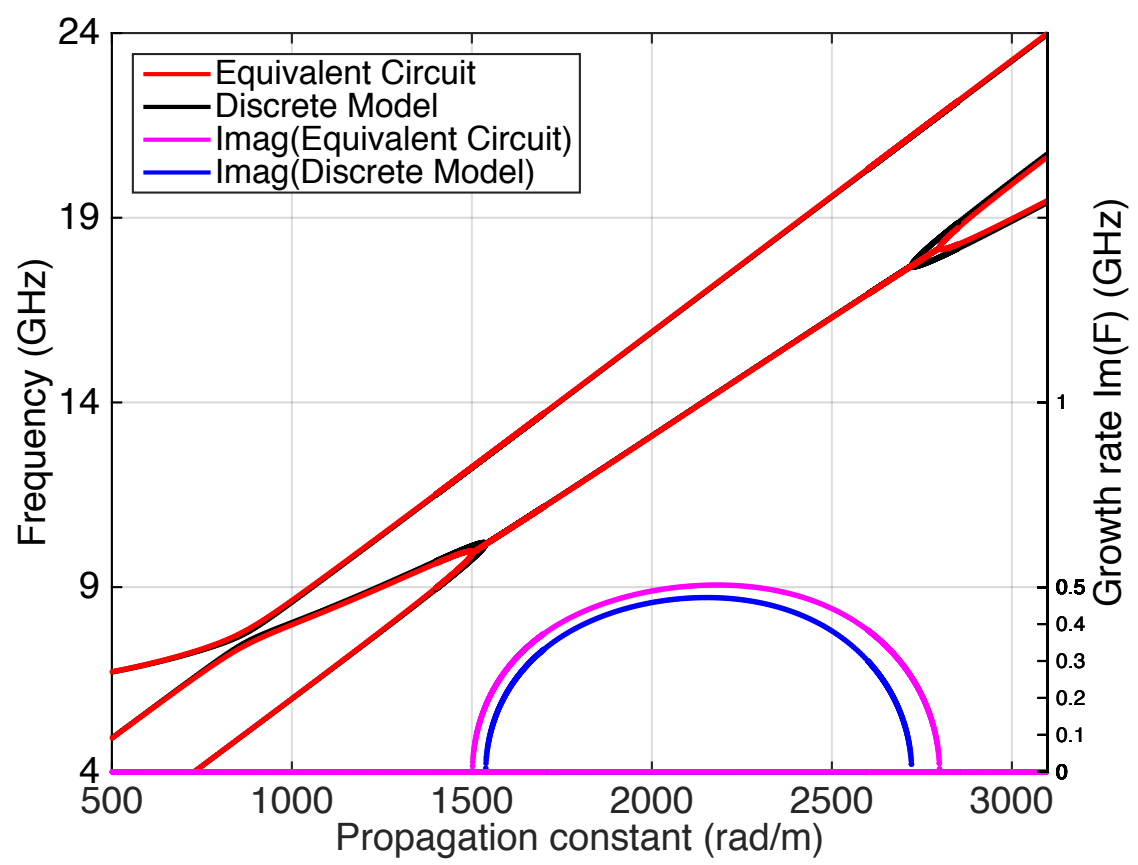

Figure 3. "Hot" linear dispersion diagrams $\mathcal{C}_{\mathrm{p}}>0$; left ordinate axis : real part of the frequency ; right axis : imaginary part. Equivalent circuit model $(20)$ is in red for the real part and in magenta for the imaginary part. Harmonic domain discrete model $(42)$ is in black for the real part and in blue for the imaginary part. Both models yield almost identical results, with fast space charge wave, slow space charge wave, and forward circuit wave, except at band edges. Tube passband from 10 to $18 \mathrm{GHz}$.

Indeed, near the wave resonance (when $\omega \simeq \Omega_{\beta}^{s}$, viz. $\beta \simeq \beta_{0}$ ), Taylor expansion yields

$$
\begin{aligned}
\omega^{2}-\beta^{2} v_{\mathrm{ph}, 0}^{2} & =2 \omega v_{\mathrm{ph}, 0}\left(\beta-\beta_{0}\right)+\cdots \\
\omega^{2}-\left(\Omega_{\beta}^{s}\right)^{2} & =2 \omega v_{\mathrm{g}}\left(\beta-\beta_{0}\right)+\cdots
\end{aligned}
$$

This approximation leads to the conclusion that the harmonic domain discrete model provides the same results as the equivalent circuit model when the dispersion diagram is a slight perturbation of the un-coupled waves, which is the case for practical devices because the Pierce parameter $\mathcal{C}_{\mathrm{p}}$ is always small.

But, outside this approximation, we expect small variations between the two models. The maximum distance between un-coupled and coupled waves occurs at the amplification band edges where mode coalescence takes place. To assess them on an example, we take the "cold" dispersion relation of a TWT and we solve the previous equation.

In (20) and (42), the propagation constant $\beta$ is treated as an independent variable and amplification is considered in time, with complex frequencies $\omega(\beta)$ whose imaginary parts are growth rates. The tube passband is defined when non-zero growth rates occur. A symbolic solver provides 

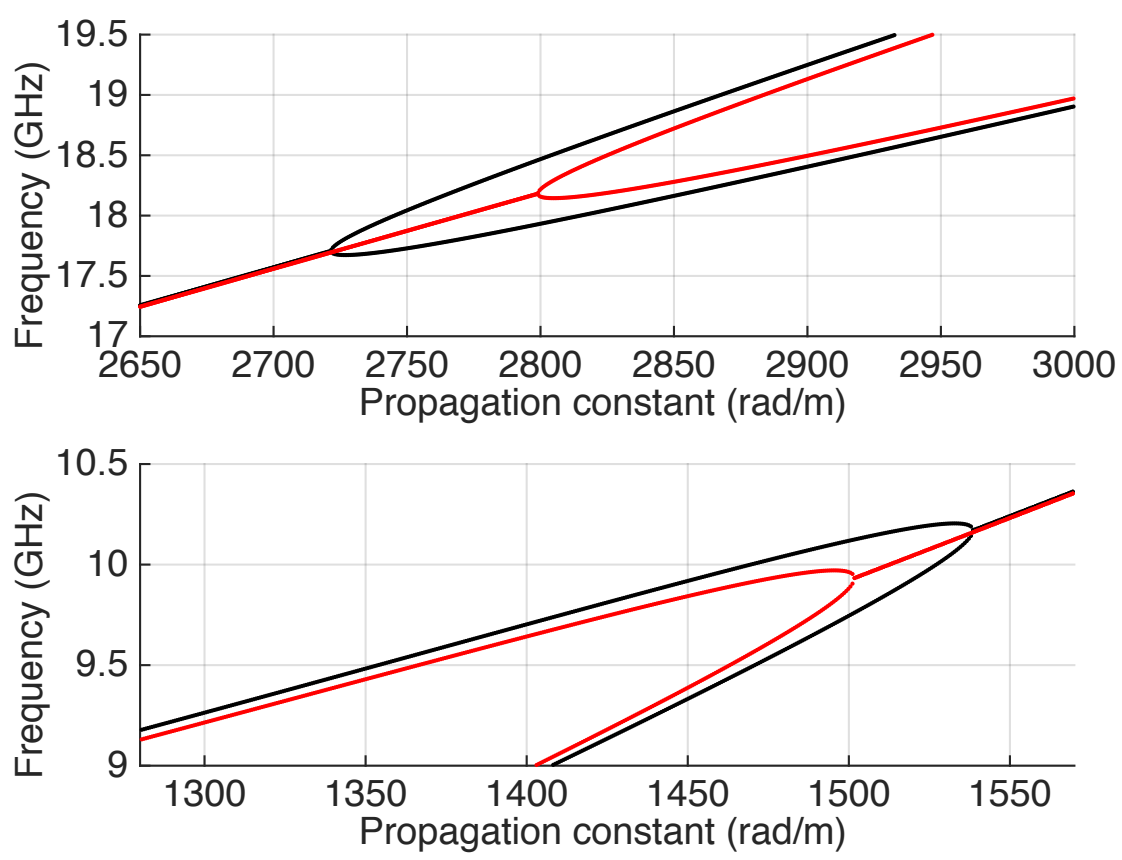

Figure 4. Zoom near band edges on "hot" linear dispersion diagrams of Fig. 3. Equivalent circuit passband starts lower in frequency, near $10 \mathrm{GHz}$, when discrete model passband starts at $10.2 \mathrm{GHz}$. Equivalent circuit passband stops higher at $18.1 \mathrm{GHz}$, when the discrete model stops at $17.7 \mathrm{GHz}$.

solutions for the four waves as displayed in Fig. 3. We immediately see the close similarity between both models as their solutions are almost superposed. The upper curve stands for the fast space charge wave, the lower curve depicts the slow space charge wave, and between them we see the forward circuit wave. The backward circuit wave, with negative frequencies or negative propagation constants, is not shown. From 10 to $18 \mathrm{GHz}$, real solutions for the slow space charge wave and the forward circuit wave are superposed, and for both waves, we have non-zero imaginary parts : this defines the passband of the tube.

A zoom at band edges of Fig. 3 is presented in Fig. 4. As expected, small differences occur in the band edge vicinity. Similar differences, but with other dispersion relations, were found in [30]. The main difference is the size of the passband, which is larger for the equivalent circuit. The difference is really small between real parts $\Re(F)$ of both dispersion relations. But, for the growth rate $\Im(F)$, the difference is more significant $(\sim 5-10 \%)$. 


\section{CONCLUSION}

We first presented another way to derive the "hot" dispersion relation of the Pierce equivalent circuit, using (less usual) beam and circuit-beam impedances, with the pedagogical intent to highlight connections between the wave-particle system and more common circuit concepts. After recalling the basis of the discrete model, we computed its "hot" dispersion relation in linear harmonic domain. Finally, an analytical comparison shows that both models lead to similar results, which validates the discrete model in small signal regime.

However, small measurable differences do exist between the models so that one of them (or both) must deviate from the experiment. We suggested elsewhere [32] that frequency-domain envelope models like Pierce's slightly violate Maxwell equations when coupling is strong. On the other hand, the only approximation in the discrete model is the truncation on the number of modes which is a sensible approximation. These arguments suggest that the Pierce model is more likely to contain approximations than the discrete model, especially because discrepancies are stronger near the band edges, where the Pierce circuit impedance tends to infinity. According to us, a major advantage of the discrete model is its validity near band edges as well as in the center of passband. We also presume that the discrete theory predicts the gain more accurately because of the significant growth rate difference between the models. But to prove this, we need to compare our model with a PIC code or with experimental measurements (this investigation is ongoing [17 [19]).

\section{Appendix A: Sheath helix approximation}

Now, we present an application of the discrete model to the sheath helix model [12], arguably the simplest three-dimensional traveling-wave tube model. Starting from the real periodic structure instead of the equivalent circuit, we obtain the circuit impedance from the tube geometry. First of all, the derivative of the normalisation (28) provides the group velocity

$$
v_{\mathrm{g}}(s, \beta)=\partial_{\beta} \Omega_{\beta}^{s}=\frac{d}{N_{\beta}^{s}} \int_{\mathcal{S}} \Re\left(\mathbf{E}_{\beta}^{s *}(\mathbf{r}) \times \mathbf{H}_{\beta}^{s}(\mathbf{r})\right) \cdot \mathbf{e}_{z} \mathrm{~d} \mathcal{S},
$$

where the surface integral is equal to $1 / d$ times the cell volume integral. In the sheath helix model, it is realistic to use only one propagation mode (so we omit superscript $s=0$ ), without space harmonics. The flux of the Poynting vector in the harmonic discrete model along the $z$-axis reads [18, 19]

$$
\langle\mathcal{P}\rangle=\frac{1}{2} \Re \frac{1}{2 \pi} \int_{-\pi}^{\pi} \tilde{\mathbf{V}}_{\beta}^{*} \tilde{\mathbf{l}}_{\beta} \frac{1}{d} \mathrm{~K}_{\beta} c^{2} \mathrm{~d}(\beta d)
$$


with the geometric propagation factor $\mathrm{K}_{\beta}$ resulting from $\mathrm{A} 1$

$$
c^{2} \mathrm{~K}_{\beta}=v_{\mathrm{g}} N_{\beta}
$$

with $c$ the celerity of light. Given the 3D boundary conditions, the solutions of the Helmholtz equations (24)-25) for $\mathbf{E}_{\beta}(\mathbf{r})$ and $\mathbf{H}_{\beta}(\mathbf{r})$ provide a definition for each eigenfield leading to

$$
\mathrm{K}_{\beta} c^{2}=\frac{\beta N_{\beta} \epsilon_{0}}{\gamma^{2}} \pi a^{2}\left|\mathrm{E}_{z, \beta}^{s}(r=0)\right|^{2} \mathcal{F}(\gamma a),
$$

with $\pi a^{2}$ the disc section area of the helix, with the so-called transverse propagation constant $\gamma=\sqrt{\beta^{2}-\left(\Omega_{\beta} / c\right)^{2}}$, and with the dimensionless impedance reduction factor $[26]$

$$
\mathcal{F}(\gamma a)=\left(1+\frac{I_{0} K_{1}}{I_{1} K_{0}}\right)\left(I_{1}^{2}-I_{0} I_{2}\right)+\left(\frac{I_{0}}{K_{0}}\right)^{2}\left(1+\frac{I_{1} K_{0}}{I_{0} K_{1}}\right)\left(K_{0} K_{2}-K_{1}^{2}\right),
$$

where $I_{m}=I_{m}(\gamma a)$ and $K_{m}=K_{m}(\gamma a)$ are modified Bessel functions of the $m^{\text {th }}$ order of the first and second kinds respectively. Using (19), we finally recover the circuit impedance in the thick $(a>0)$ beam model as

$$
Z_{\mathrm{c}}(\beta)=\frac{1}{\pi a^{2} \epsilon_{0}} \frac{\gamma^{2}}{\Omega_{\beta} \beta^{3}}\left[\mathcal{F}\left(\gamma_{0} a\right)\right]^{-1}
$$

resulting from the helix geometry [12, 33]. A similar development can be done for any tube geometries from the discrete model, so it is well adapted to investigating 3D structures.

\section{Appendix B: Beam-plasma systems}

Because they generate only little noise, traveling-wave tubes have also proved to be good tools for plasma physics (besides the fact that the beam is already a plasma). In the classic beam-plasma system [34 37], waves are propagated using the classic plasma itself. Longitudinal electrostatic (or Langmuir) waves in the plasma have phase velocities on the scale of a few times the particles thermal velocity [24].

To study this system, we substitute the propagating medium with a slow-wave structure like in a TWT. Following [35], considering the power definition, in harmonic domain, for a one-dimensional plasma $\langle\mathcal{P}\rangle=\int v_{\mathrm{g}} \mathcal{E} \mathrm{d} \mathcal{S}$, where $v_{\mathrm{g}}$ is the group velocity, and $\mathcal{E}$ is the wave energy density of the plasma given by

$$
\mathcal{E}=\left.\frac{\epsilon_{0}}{2} \omega \frac{\partial}{\partial \omega}(\varepsilon(\beta, \omega))\right|_{\omega, \beta_{0}}\left\langle E_{z, \mathrm{c}}^{2}\right\rangle
$$

from the average squared electric field, with $\varepsilon(\beta, \omega)$ the plasma dielectric function. Thus

$$
\langle\mathcal{P}\rangle=\left.\frac{-\pi a^{2} \epsilon_{0}}{2} \omega \frac{\partial}{\partial \beta}(\varepsilon(\beta, \omega))\right|_{\beta_{0}}\left|E_{z, \mathrm{c}}\right|^{2},
$$


because $v_{\mathrm{g}}=\frac{\partial \omega}{\partial \beta}$. Using $(19)$, we make the link between the beam-plasma system and the beamslow-wave structure system by identifying the plasma impedance

$$
Z_{\mathrm{p}}=\frac{-1}{\pi a^{2} \epsilon_{0}} \frac{2}{\omega \beta^{2}}\left[\left.\frac{\partial}{\partial \beta}(\epsilon(\beta, \omega))\right|_{\beta_{0}}\right]^{-1}
$$

with $Z_{\mathrm{c}}$. Eq. (B3) can also be obtained from computing circuit potentials of both systems, as done in [38]. Then the Pierce parameter is given by

$$
\mathcal{C}_{\mathrm{p}}^{3}=\frac{-\omega_{\mathrm{p}}^{2}}{\omega \beta^{2} v_{0}}\left[\left.\frac{\partial}{\partial \beta}(\epsilon(\beta, \omega))\right|_{\beta_{0}}\right]^{-1} .
$$

We immediately see the analogy between the helix slow-wave structure circuit impedance A6, depending on the tube geometry, and the plasma impedance (B3), depending on the plasma dielectric function. It is because waves in a TWT are expressed thanks to the dispersion relation and the way they are coupled with the beam. Ref. 34] takes $\beta=\beta_{\mathrm{e}}=\omega / v_{0}$, so the linear Landau growth rate is $\gamma_{\max }=\left(n_{\mathrm{p}} / n_{\mathrm{b}}\right)^{1 / 3} \sqrt{3} \mathcal{C}_{\mathrm{p}} \omega / 2^{1 / 3}$, with $n_{\mathrm{b}}$ and $n_{\mathrm{p}}$ the beam and plasma densities. Analogy of the beam-plasma and the TWT slow-wave structure is allowed by replacing the dielectric function with the geometric factor contained in the circuit impedance.

\section{ACKNOWLEDGEMENT}

The authors are pleased to thank F. Doveil for his suggestions. DM and YE are grateful to A. Figotin for fruitful comments on a previous version of this work. NMR and AGT acknowledge support from the Russian Science Foundation grant No 17-12-01160. AGT benefited from an Ostrogradski fellowship from the French embassy in Russia.

[1] Spohn H 2004 Dynamics of charged particles and their radiation field (Cambridge university press, Cambridge)

[2] Rohrlich F 2007 Classical charged particles (World Scientific, Singapore)

[3] Bénisti D 2016 Envelope equation for the linear and nonlinear propagation of an electron plasma wave, including the effects of Landau damping, trapping, plasma inhomogeneity, and the change in the state of wave Phys Plasmas 23102105 (err 24 019901)

[4] Elskens Y and Escande D 2002 Microscopic dynamics of plasmas and chaos (IoP Publishing, Bristol)

[5] Bonifacio R, Casagrande F, Cerchioni G, De Salvo Souza L, Pierini P and Piovella N 1990. Physics of the high-gain FEL and superradiance Riv Nuovo Cim 13 1-69

[6] Doveil F and Macor A 2006 Phys. Plasmas 13055704 
[7] Doveil F, Elskens Y and Minenna D F G 2018 Wave-Particle Interaction studied in a Traveling Wave Tube ICPP 2018 (Vancouver)

[8] Davidson R C and Qin H 2001 Physics of intense charged particle beams in high energy accelerators (Imperial College press, London)

[9] Eichmeier J A and Thumm M (eds) 2008 Vacuum electronics - Components and devices (Springer, Berlin)

[10] Carter R G 2018 Microwave and RF vacuum electronic power sources (Cambridge univ. press, Cambridge)

[11] Minenna D F G et al 2018 The traveling-wave tube in the history of telecommunication, to be published

[12] Pierce J R 1950 Traveling wave tubes (Van Nostrand, New York)

[13] Gilmour A S Jr 1994 Principles of traveling wave tubes (Artech House, Boston)

[14] Kuznetsov S P 1980 On one form of excitation equations of a periodic waveguide Sov J Commun Technol Electron 25 419-421

[15] Ryskin N M, Titov V N and Yakovlev A V 2009 Nonstationary nonlinear discrete model of a coupledcavity traveling-wave-tube amplifier IEEE Trans Electron Devices $\mathbf{5 6} 928-934$

[16] André F, Bernardi P, Ryskin N M, Doveil F and Elskens Y 2013 Hamiltonian description of selfconsistent wave-particle dynamics in a periodic structure Europhys Lett 10328004 (5 pp)

[17] Terentyuk A G, Rozhnev A G and Ryskin N M 2017 Discrete model of a folded-waveguide travelingwave tube IVEC 2017 (London)

[18] Minenna D F G, Elskens Y and André F 2017 Electron-wave momentum exchange and time domain simulations applied to traveling wave tubes IVEC 2017 (London)

[19] Minenna D F G, Elskens Y, André F and Doveil F 2018 Electromagnetic power and momentum in $N$-body hamiltonian approach to wave-particle dynamics in a periodic structure, to be published

[20] André F, Théveny S, Doveil F and Elskens Y 2015 First comparison of new TWT discrete model with existing models IVEC 2015 (Beijing)

[21] Kuznetsov A P, Kuznetsov S P, Rozhnev A G, Blokhina E V and Bulgakova L V 2004 Radiophys Quantum Electron $\mathbf{4 7} 356-373$

[22] Louisell W H 1960 Coupled mode and parametric electronics (Wiley, New York)

[23] Hertz H R 1889 Die Kräfte electrischer Schwingungen, behandelt nach der Maxwell'schen Theorie Wied Ann 36 1-22

[24] Bohm D and Gross E P 1949 Theory of plasma oscillations. A. Origin of medium-like behavior Phys Rev 75(12) 1851-1864

[25] Simon D H, Wong P, Chernin D, Lau Y Y, Hoff B, Zhang P, Dong C F and Gilgenbach R M 2017 On the evaluation of Pierce parameters $C$ and $Q$ in a traveling wave tube Phys Plasmas $\mathbf{2 4} 033114$

[26] Pierce J R 1947 Theory of the beam-type traveling-wave tube Proc IRE $\mathbf{3 5}$ 111-123

[27] Bernardi P, André F, David J-F, Le Clair A and Doveil F 2011 Efficient Time-Domain Simulations of a Helix Traveling-Wave Tube IEEE Trans Electron Devices $\mathbf{5 8}$ 1761-1767 
[28] Bernardi P, André F, David J-F, Le Clair A and Doveil F 2011 Control of the reflections at the terminations of a slow wave structure in the nonstationary discrete theory of excitation of a periodic waveguide IEEE Trans Electron Devices $\mathbf{5 8}$ 4093-4097

[29] Aïssi A 2008 La modélisation des tubes à onde progressive à hélice en domaine temporel $\mathrm{Ph} . \mathrm{D}$. thesis (Univ. Provence, Marseille) tel-00406576.

[30] Théveny S 2016 Approches fréquentielle et temporelle de la dynamique des tubes à ondes progressives Ph.D. thesis (Aix-Marseille Univ., Marseille)

[31] Gel'fand I M 1950 Eigenfunction expansions for an equation with periodic coefficients Dokl Akad Nauk SSSR 73 1117-1120.

[32] Théveny S, André F and Elskens Y 2016 On frequency and time domain models of traveling wave tubes, preprint hal-01340471

[33] Tien P K 1953 Traveling-wave tube helix impedance Proc IRE 41 1617-1623

[34] O'Neil T M, Winfrey J H and Malmberg J H 1971 Nonlinear interaction of a small cold beam and a plasma Phys Fluids 14 1204-1212

[35] Tsunoda S I 1982 Wave enhancement due to a static electric field Ph.D. thesis (Univ. California at San Diego, La Jolla, California)

[36] Tsunoda S I and Malmberg J H 1982 Effect of a static electric field on the trapping of beam electrons in a slow wave structure Phys Rev Lett 49 546-549

[37] Doveil F, Macor A and Aïssi A 2007 Observation of hamiltonian chaos and its control in wave particle interaction Plasma Phys Control Fusion 49 125-135

[38] Guyomarc'h D 1996 Un tube à onde progressive pour l'étude de la turbulence plasma Ph.D. thesis (Univ. Provence, Marseille) 